\title{
Extreme Light Infrastructure Nuclear Physics (ELI-NP): Present status and perspectives
}

\author{
N.V. ZAMFIR
}

ELI-NP, "Horia Hulubei" National Institute for Physics and Nuclear Engineering (IFIN-HH), 30 Reactorului Street, Bucharest-Măgurele, 077125 Romania

\begin{abstract}
Extreme Light Infrastructure - Nuclear Physics (ELI-NP), a new Research Center under construction, will use extreme electromagnetic fields for nuclear physics research and will be operational in 2018. The status of the Project implementation will be presented. At ELI-NP, a high power laser system together with a very brilliant gamma beam are the two main research tools. Their targeted operational parameters will be described. The related experimental set-ups will be presented, together with the main directions of the research envisioned.
\end{abstract}

\section{Introduction}

The Extreme Light Infrastructure (ELI), listed on the ESFRI (European Strategic Forum for Research Infrastructures) 2006 road-map, is distributed among three European countries (the Czech Republic, Hungary and Romania) and funded by European Commission through Structural Funds. The Extreme Light Infrastructure - Nuclear Physics (ELI-NP) pillar [1], located near Bucharest in the Magurele Physics research campus, is midway the implementation phase. The project was approved by European Commission in September 2012 and will be operational in 2018. The civil construction of the new center, consisting of laser building, accelerator and experiments 
building, laboratories building, offices, guest house and canteen started in June 2013 and will be finished in the beginning of 2016 .

Figure 1 shows the status of the construction.

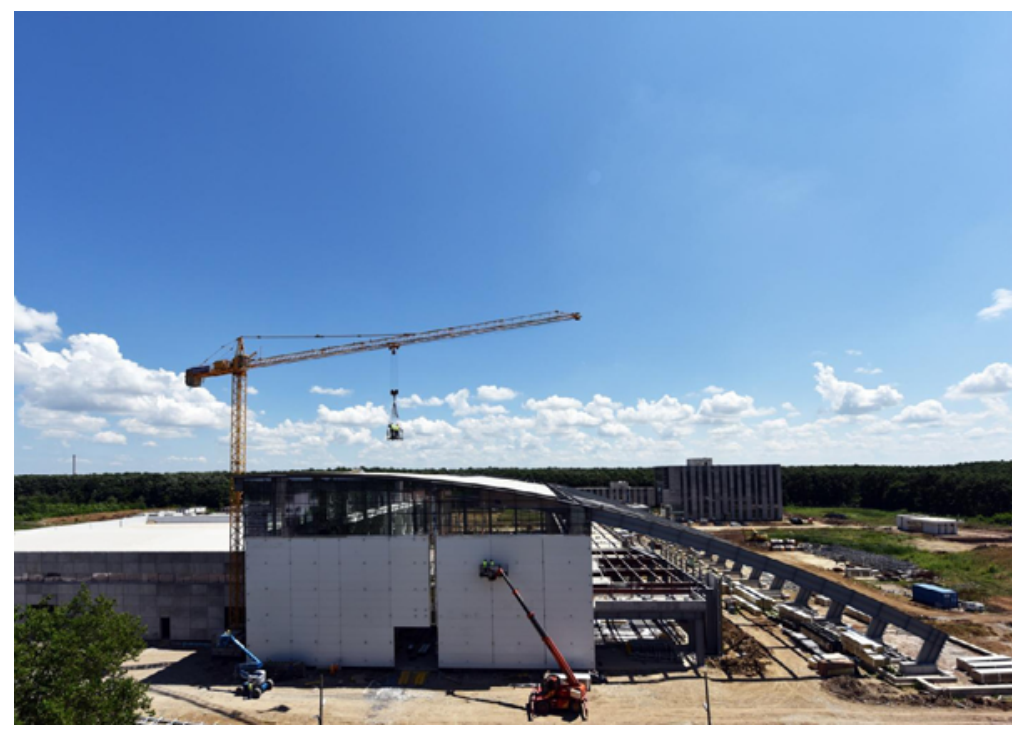

Figure 1: The civil construction stage of ELI-NP in May 2015.

The total cost of the facility will be 300 million euro, without VAT.

At the time of entering operation, ELI-NP will be the most advanced research infrastructure in the world focused on photonuclear physics studies and applications. The facility will cover frontier fundamental physics, new nuclear physics and astrophysics as well as applications in nuclear materials and radioactive waste management, materials science and life sciences [2-4].

\section{Scientific equipment}

Two large, cutting-edge pieces of equipment will promote ELI-NP to the forefront of scientific research at international level: the High Power Laser System (HPLS) and the high intensity Gamma Beam System (GBS).

The High Power Laser System [5] consists of 2 arms of 10 PW each based on Optical Parametric Chirped Pulse Amplification (OPCPA) system $[6,7]$ at about $820 \mathrm{~nm}$ central wavelength, which includes Ti: Sapphire amplifiers to bring the final output energy to the level of a few hundreds of Joule. Subsequently, the pulses are compressed to around a 20 fs pulse duration. The repetition rate is 1 shot per min for each of the two arms. Along 
the two amplification chains, additional outputs with corresponding optical compressors will be installed. Their corresponding power levels are $0.1 \mathrm{PW}$ and $1 \mathrm{PW}$ at repetition rates of $10 \mathrm{~Hz}$ and $1 \mathrm{~Hz}$, respectively. The highpower laser system of ELI-NP will be constructed by an association between Thales Optronique France and Thales Romania.

The Gamma Beam System will produce brilliant Gamma ray beam, with energy tuneable up to $20 \mathrm{MeV}$, obtained by back-scattering of optical photons on electrons from a warm LINAC with energies up to $720 \mathrm{MeV}[8,9]$. The very low cross section for Compton scattering will be compensated in obtaining high brilliance gamma beam by very intense high repetition rate photon beam, very intense low emittance electron beam and a very small and precise interaction volume.

The output specifications for the Gamma beam (see Table 1) were established in a series of workshops and meetings organized with the scientific community interested in ELI-NP, in order to satisfy the needs of the scientists for the progress of their research but also to have realistic expectancies that are technically feasible within the timeframe of project implementation. The main characteristics of the gamma beam that will make it unique will be the very narrow relative bandwidth (of the order of $10^{-3}$ ), one order of magnitude better than present-day machines, and the extremely high brilliance - at least two orders of magnitude better than present state-of-the-art machines. Consequently, very good energy level selection capabilities and reduced irradiation times will be possible in experiments, making possible experiments that were not feasible before.

Table 1: Output parameters for the gamma beam system

\begin{tabular}{ll}
\hline Type & Range \\
\hline Photon energy $[\mathrm{MeV}]$ & $0.2-19.5$ \\
Divergence $[\mu$ Rad] & $25-200$ \\
Average Bandwidth & $\leq 5 \cdot 10^{-3}$ \\
Nr. Photon/s & $<2.6 \cdot 10^{5}$ \\
within FWHM bdw $[1 /(\mathrm{s} \mathrm{eV})]$ & $10^{20}-10^{23}$ \\
Peak Brilliance $\left[\mathrm{N}_{\mathrm{ph}} / \mathrm{s} \cdot \mathrm{mm}^{2} \cdot \mathrm{mrad}^{2} \cdot 0.1 \%\right]$ & $\geq 100$ \\
Minimum Frequency $\gamma$ Ray Macropulses $[\mathrm{Hz}]$ & \\
\hline
\end{tabular}

The Gamma Beam System will have two gamma-ray outputs available for use independently, one for the lower part of the energy range (up to approximately $3.5 \mathrm{MeV}$ ) obtained using the first half of the accelerator, and 
a second one for higher energies (up to approximately $19.5 \mathrm{MeV}$ ).

The gamma beam system will be constructed by EuroGammas, a European consortium led by INFN Italy and composed of leading research institutions and private companies in accelerator and laser technologies from 8 European countries.

\section{Experimental areas}

The scientific case for ELI-NP was elaborated by an international collaboration of more than 100 scientists from 30 countries and published as the ELI-NP White Book [2]. It is based on the unique features of the highpower laser and gamma beams. The main research topics of interest are: laser driven nuclear physics experiments, characterization of the laser-target interaction by the means of nuclear physics methods, photonuclear reactions, exotic nuclear physics and astrophysics. In addition to fundamental themes, applications of HPLS and GBS are under study. Radiation induced damage and gamma induced nuclear reactions are major research area in nuclear engineering. Their applications extend from nuclear power plants to medicine and from space science to material science.

The ELI-NP team together with their collaborators from the international scientific community shaped the future scientific program of ELI-NP is a series of workshops and defined ten development directions for the facility. The Technical Design Reports (TDRs) are finalized and approved by ELI-NP International Scientific Advisory Board in June 2015.

ELI-NP Center will have eight experimental rooms:

- E1: laser induced nuclear reactions;

- E2: nuclear resonance fluorescence and applications;

- E3: positrons source;

- E4 / E5: accelerated particle beams induced by HPLS (0.1/1 PW) at high repetition rates;

- E6: intense electron and gamma beams induced by high power laser beams;

- E7: experiments with combined laser and gamma beams;

- E8: nuclear reactions induced by high energy gamma beams. 


\section{Experiments}

\subsection{Laser-based experiments}

The powerful lasers will be used to obtain accelerated ions. The acceleration of particles using lasers can occur following several mechanisms, depending on the characteristics of the incident beam and target: Wake-Field acceleration [10] for gas targets, Target Normal Sheath Acceleration (TNSA) [11] for solid targets or Radiation Pressure Acceleration (RPA) [12] for much thinner (nanometers) foil targets. The acceleration of particles using laser beams is of great interest due to many applications it may trigger in a number of fields, from medicine (compact and more versatile accelerators for ion-therapy) to management of nuclear waste.

The study of the interaction of radiation with matter by means of nuclear physics techniques is one of the new areas where ELI-NP will constitute a big step forward with respect to existing facilities. Interaction of the high power $(\mathrm{PW})$ laser radiation with the solid state matter produces specific effects, not completely known and/or understood. The detailed knowledge of these effects has a fundamental interest for understanding the behaviour of various materials in extreme conditions of irradiation. The study of the irradiation effects significantly helps to optimization of the materials and components operating in high radiation fields.

One of the experiments proposed aims to the production of neutron-rich nuclei around the $\mathrm{N}=126$ waiting point of the r-process via the fission-fusion reaction mechanism [13].

\subsection{Experiments based on the gamma beam}

The state-of-the-art characteristics of the gamma beam will make feasibly the study of detailed structure of dipole states in nuclei via Nuclear Resonance Fluorescence [14], including the fine structure of Giant Dipole Resonances (GDR) and Pigmy Dipole Resonances. The fine structure of fission barrier will be also studied. Compared to former gamma-ray facilities, the much improved bandwidth is decisive for this new facility, because an important factor in this type of experiments is the energy bandwidth of the beam, which should be smaller than the normal energy gap between nuclear levels. Several experiments, like the parity violation experiment, only become possible also due to this much better bandwidth.

Laboratory astrophysics experiments aiming at explaining the nucleosynthesis processes will be possible, through direct or inverse reactions, i.e. through $(\gamma, \mathrm{n}),(\gamma, \mathrm{p})$ or $(\gamma, \alpha)$ reactions. Reactions relevant for the $\mathrm{p}-$ and 
r-processes will be investigated, to advance the explanation of the formation of a large part of the known elements in the Universe.

The gamma beam can be used to study new techniques to map the isotope distributions of nuclear materials or radioactive waste remotely via Nuclear Resonance Fluorescence (NRF) measurements. At lower energies, around $100 \mathrm{keV}$ the high resolution of the beam is very important for protein structural analysis. In addition it will be produced low energy, brilliant, intense neutron and positron beams, which will open new fields in materials science and life sciences.

The new production schemes of medical isotopes [15] (e.g., ${ }^{99} \mathrm{Mo}-$ currently used in therapies, ${ }^{195 \mathrm{~m}} \mathrm{Pt}$ - nuclear imaging to determine efficiency of chemotherapy, ${ }^{117 \mathrm{~m}} \mathrm{Sn}$ - emitter of low energy Auger electrons for tumour therapy) via $(\gamma, \mathrm{n})$ processes may also reach socio-economical relevance.

Applied research and development will be enabled by the very high degree of collimation and the intensity of the gamma beam, allowing studies in materials science. A relevant example is the investigation of methods new methods to produce thermal neutrons, through photonuclear reactions $(\gamma, \mathrm{n})$. Another example would be the creation of an intense positron sources by means of the $\left(\gamma, \mathrm{e}^{+} \mathrm{e}^{-}\right)$reaction $[16]$.

\subsection{Experiments employing lasers and gamma beam}

The possibility to study fundamental processes with very brilliant optical and gamma beams will be unique and shall advance science much faster. The proposals for future experiments include two main topics with staged developments [17]. The first one is linked to the possibility to reach at ELI-NP conditions similar to the interior of stars and production and photoexcitations of isomers can be studied. The second topic is probing the photon-photon interactions at low energy (below $\mathrm{MeV}$ ), which have not been explored to date, by using the laser-laser,laser-gamma, and gammagamma collisions. By these various combinations can be tested nonlinear QED effects. It also includes the pair production via the tunnelling process in extremely high-intensity laser fields. The laser-electron experiments aims at the generation of polarized gamma-rays from sub- $\mathrm{GeV}$ to $\mathrm{GeV}$ as probes for the later stage experiments to explore the vacuum birefringence under high-intensity laser fields. 


\section{Conclusions}

The ELI-NP project is the result of a pan-European collaborative effort that extended beyond the borders of the European Union. Research communities in more than 20 countries actively contributed to the definition of the project. The present report is a short, updated synthesis of these efforts.

ELI-NP will host two cutting edge infrastructures, namely $2 \times 10 \mathrm{PW}$ laser systems and a brilliant gamma beam. They will open new directions in fundamental research. They will allow also applied research and development in unique conditions, relevant also for companies acting in the fields of medicine (radionuclides and hadron-therapy), telecommunications (materials in high intensity radiation fields), engineering industry (non-destructive testing), security (scanners based on nuclear resonance fluorescence of sensitive nuclear materials).

\section{Acknowledgments}

The ELI-NP project is the result of an international collaborative effort of more than 100 scientists from 20 countries and their contribution to the definition and implementation of the project scientific program is gratefully acknowledged. The tremendous work of the ELI-NP scientific team and the essential contribution of my enthusiastic and tenacious colleagues from the Management team in the implementation of the project are also deeply acknowledged. The ELI-NP project is co-financed by the Romanian Government and European Union through the European Regional Development Fund.

\section{References}

[1] www.eli-np.ro

[2] ELI-NP White Book eds. Habs D. et al. (2010), http://www.elinp.ro/documents/ELI-NP-WhiteBook.pdf

[3] Zamfir N.V., EPJ Web of Conferences 66 (2014) 11043

[4] Zamfir N.V., EPJ Special Topics 223 (2014) 1221

[5] Ursescu D. et al., Proc. of SPIE, 87801H-1 (2013)

[6] Strickland D., Mourou G., Opt.Comm. 56 (1985) 219 
[7] Ross I.N. et al., Opt.Commun. 144 (1997) 125

[8] Adriani O. et al., Technical Design Report; EuroGammaS proposal for the ELI-NP Gamma beam System, arXiv:1407.3669v1 [physics.acc-ph].

[9] Ur C. A. et al., Acta Physica Polonica B46 (2015) 743

[10] Tajima T. and Downson J.M., Physical Review Letters 43 (1979) 267

[11] Wilks S.C. et al., Phys. Plasma 8 (2001) 542

[12] Henig A. et al. , Phys. Rev. Lett. 103 (2009) 245003

[13] Thirolf P.G. et al., EPJ Web of Conferences 38 (2012) 08001

[14] Ulrich Kneissl, Norbert Pietralla, Andreas Zilges, J. Phys. G: Nucl. Part. Phys. 32 (2006) R217

[15] Habs D., Köster U., Applied Physics B103 (2011) 501

[16] Mills A.P. and Gullikson E.M., Appl. Phys. Lett. 49 (1986) 1121

[17] TDR for combined laser gamma experiments at ELI-NP, eds. Kensuke Homma, Keita Sato, Ovidiu Tesileanu (2015) 